\title{
INDOCYANINE GREEN VIDEOANGIOGRAPHY USing COOLED CHARge-COUPLED DeVices in Central Serous CHOROIDOPATHY
}

\author{
C. Ciamberlini, ${ }^{\dagger}$ V. Guarnieri, ${ }^{\dagger}$ Giuseppe Longobardi, $^{\dagger}$ Pasquale Poggi, ${ }^{\dagger}$ M. C. Donati, \\ and G. Panzardi ${ }^{\ddagger}$ \\ ${ }^{\dagger}$ Istituto Nazionale di Ottica, L. go E. Fermi, 6, Firenze 50125, Italy; ${ }^{\dagger}$ Il Clinica Oculistica \\ Universita' di Firenze, V. le Morgagni, 85, Firenze 50141, Italy \\ (Paper JBO-079 received Feb. 21, 1996; revised manuscript received Jan. 6 1997; accepted for publication Feb. 25, 1997.)
}

\begin{abstract}
In order to evaluate the use of low level light (LLL) indocyanine green angiography in the diagnosis and treatment of central serous choroidopathy (CSC), a new system for LLL infrared videoangiography is described. The system was coupled with a standard fundus camera employing a low-power continuous light source. The system consists of a cooled charge-coupled device (CCD) camera and a third-generation image intensifier. The CCD camera investigates the pathologies of choroidal images with very good performance in terms of spatial resolution, dynamic range, and signal-to-noise ratio. The image intensifier carries out the choroidal angiography in real time. The results of 48 cases of CSC demonstrated the following ICGV features: focal RPE leakage; hyperfluorescent areas appearing in late phases and underlying the focal leakage or pigment epithelium detachment (PED); PED in the guise of early hyperfluorescent areas; delays in choroidal filling. The findings support the hypothesis that CSC originates in the choroid with localized hyperpermeability. The system described minimized retinal illumination and demonstrated increased angiographic resolution and contrast compared with conventional ICG angiographic systems. ( 1997 Society of Photo-Optical Instrumentation Engineers. [S1083-3668(97)00502-9]
\end{abstract}

Keywords low level light; central serous choroidopathy (CSC); indocyanine green (ICG) videoangiography; image intensifier.

\section{INTRODUCTION}

Retinal and choroidal angiography represents an important diagnostic procedure in ophthalmology. It exploits the fluorescence and absorption properties of fluorescein and indocyanine green (ICG). Angiography using dyes was developed to take advantage of their unique fluorescence and absorption properties to study important eye diseases in vivo. ${ }^{1}$ Indocyanine green dye was used in fluorescence infrared angiography (absorption at $805 \mathrm{~nm}$ and emission at $830 \mathrm{~nm}$ ) to investigate choroidal circulation.

Fundus cameras are found in retinal and choroidal angiographic practice. The standard fundus camera includes a system for recording the eye fundus by photography or by infrared TV camera. The accuracy of techniques using fluorescent dyes as markers depends on both the imaging optical path and the illumination system. The intensity of the excitation source must be within acceptable physiologic limits in order to avoid retinal damage and

Address all correspondence to Giuseppe Longobardi. E-mail: ceo@fox.ino.it. patient discomfort. Choroidal angiogram imaging involves some complex problems because of the relatively low level of ICG fluorescence intensity, the anatomy of the ocular fundus, and the rapid transit of ICG through the choroidal vessels. The images are sent to either a film or a charge-coupled device (CCD). Several two-dimensional detectors are under investigation for angiographic imaging: charge-coupled devices and intensified TV cameras are capable of performing high fundus imaging and fluorescein angiography (FA) at very low light levels. ${ }^{2}$ Rapid developments in both CCD and image intensifier tubes in terms of sensitivity, resolution, and cost reduction are making these detectors more and more attractive for videoangiography applications.

In this paper we describe an infrared videoangiography system using both a CCD-cooled camera and an intensified TV camera capable of investigating the structures and pathologies of the choroid; furthermore, we report our clinical results in the

1083-3668/97/\$10.00 @ 1997 SPIE 
study of a specific choroidal pathology, central serous choroidopathy (CSC).

\section{SAFETY CONSIDERATIONS AND RISKS TO PATIENTS}

In dealing with the development of ophthalmic instrumentation, a fundamental problem to be considered is the hazard for the eye caused by the intense light. It is well known that in the visible and near-infrared (NIR) range the anatomical structures of the eye behave differently with respect to incident radiation. Furthermore, the response of the eye greatly depends on the nature of the radiation itself. Generally speaking, a coherent light is more dangerous than an incoherent light.

At present, the recommended maximum amounts of incoherent light are given in threshold limit values (TLV) issued by the American Conference of Governmental and Industrial Hygienists (ACGIH). These guidelines are periodically revised to reflect new knowledge about the effects of radiation on tissue. ${ }^{3,4}$

The spectral range of this device is within the IR-A range (700 to $1400 \mathrm{~nm}$ ), in which the main injury mechanism for the eye is photothermic: excess energy, due to photon radiation, is dissipated and increases the temperature of the organic tissue, generating a structural alteration of the substrate.

For usual angiographic examination conditions, we can assume a total exposure time of not less than $20 \mathrm{~min}$. This time comprises the early phases of the process, in which the fluorescence is stronger, but also the later ones where the intensity being lower, a power increase or, alternatively, integration time is often needed.

At the wavelengths considered and taking into account the actual conditions of the examination, the TLV suggested by the ACGIH is $10 \mathrm{~mW} / \mathrm{cm}^{2}$ on the anterior surface of the eye. The incident light power of the lighting source employed in our system is about 40 times less than this limit. Thus it is possible to conclude that there are no risks for the eye during the whole examination process because the system works back to the exposure limit suggested by the ACGIH.

\section{SYSTEM SETUP}

The system developed for this study can be easily incorporated in every standard fundus camera. The essential optoelectronic devices of the compound fundus camera modified to work at very low light levels are illustrated in Figure 1. For convenience these components may be grouped into two subsystems, namely, lighting and image detecting. The former uses a low-voltage halogen lamp as its light source and is described in the next section; the latter includes two channels as specified in Section 3.2.

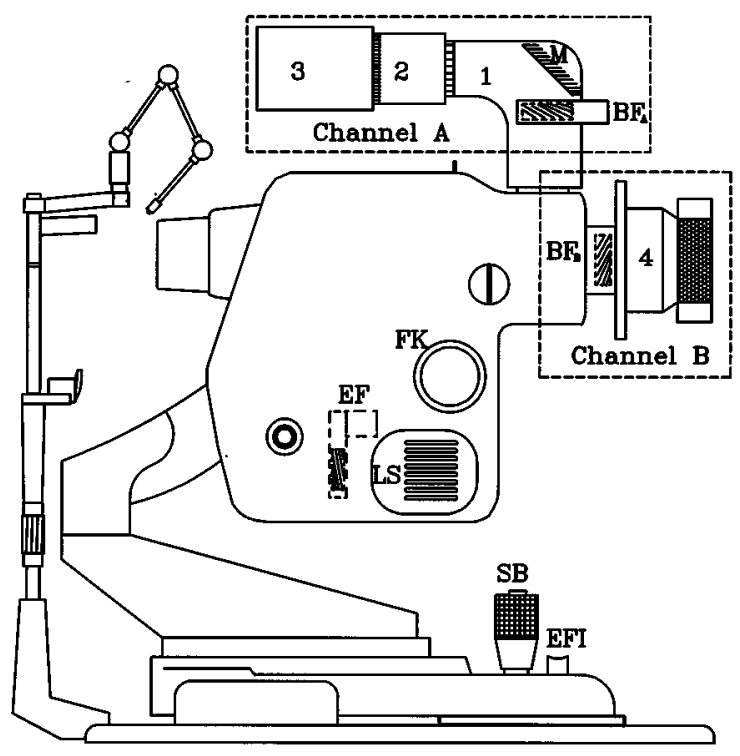

Fig. 1 Schematic view of system setup. BF, barrier filter; 1 , optical transducer; 2, HOT third-generation image intensifier; $M$, mirror; 3, intensified CCD camera; 4, cooled camera; FK, focusing knob; $E F$, exciting filter; LS, light source; SB, shutter button; EFI, exciting filter insertion.

\subsection{LIGHTING SYSTEM}

The fundus images are taken with very low intensity light that crosses an 805-nm exciting filter. A barrier filter at $830 \mathrm{~nm}$ at the input of the detecting system is used to separate fluorescence from lighting.

The ICG angiograms shown in this paper were recorded using a 50-W incandescent continuous source behind the illuminator condenser lens. Light intensity can be adjusted because the overall quality of the image is directly related to the amount of light projected into the well-dilated pupil of the eye. In the event of the patient's pupil not dilating well, the intensity lamp was increased to compensate the underexposed picture. The rise in lamp output was also carried out for late-phase image recording.

During ICG choroidal videoangiography, the maximum measured incident irradiance was $300 \mu \mathrm{W} / \mathrm{cm}^{2}$ on the anterior segment and thus $255 \mu \mathrm{W} / \mathrm{cm}^{2}$ on the retina. ${ }^{5}$ All conventional ICGbased fundus cameras employ a double light source: a 100-W halogen lamp for observation and a $300-$ to $500-\mathrm{W}$ flash lamp for imaging. The system described here performs the two steps with only a 50-W observation lamp.

Apart from eye damage hazards, the use of nearinfrared low level light avoids patient light stress even during the focusing operation before ICG injection. Reflection losses that inevitably result from misalignment due to manufacturing and assembly tolerances are reduced by coating the surfaces of the optical components within $805 \mathrm{~nm}$ in the light- 
ing pathway of illumination and within $830 \mathrm{~nm}$ in that of fluorescence imaging.

\subsection{IMAGE DETECTOR SYSTEM}

The detector system developed for this study consists of two channels (see Figure 1). The first one, channel $\mathrm{A}$, is composed of a series of three devices housed in a compact cylindrical black anodized head: an optical-optical transducer (1), and image intensifier (2), and an intensified CCD camera (3). Channel $\mathrm{B}$ includes a barrier filter $\left(\mathrm{BF}_{\mathrm{B}}\right)$ aligned with a cooled CCD camera (4).

Channel A is composed of a collimating lens with a viewing angle that covers a fundus area of $50 \mathrm{deg}$, an interferential removable barrier filter $\left(\mathrm{BF}_{\mathrm{A}}\right)$, a plane mirror $(\mathrm{M})$, and an optical system that reduces the eye fundus image size from 27 to $18 \mathrm{~mm}$. This corresponds to the diameter size of the thirdgeneration high output (HOT) image intensifier photocathode (2), which is directly connected to the output of the optical-optical transducer. An intensified CCD camera (3) is inserted in line with the HOT device. This latter is a low level light, highresolution camera with intensifier gain. By using a high-performance first-generation image intensifier, image quality is improved by coupling the intensifier to the CCD by means of an optical fiber plate. The use of coherent fiber optics increases the optical efficiency, which is significantly greater than can ever be achieved using lenses for coupling. In addition, when a change in image scale is needed, as in the system used from $18 \mathrm{~mm}$ of the intensifier to 11 $\mathrm{mm}$ of the diagonal of the CCD, fiber optics coupling is an efficient way to achieve it.

Our emphasis is on higher definition or resolving power, requiring more detail for images. To increase image details, greater low level light resolution is required. This is strongly dependent on pixel density and signal to noise (S/N) ratio. Low level light resolution of image intensifier tubes is limited by the photoemission statistics, which depend on photocathode sensitivity. The third-generation image intensifier tube uses a high photosensitivity GaAs photocathode with a responsivity of 150 $\mathrm{mA} / \mathrm{W}$ at $830 \mathrm{~nm}$, which is the emission wavelength of the ICG tracer. The resolution limit is 52 $\mathrm{lp} / \mathrm{mm}$ and the gain is $8 \times 10^{4}$.

The CCD sensor is a line transfer device with 753 active horizontal pixels per line and 576 vertical lines. The limiting resolution is $620 \mathrm{TV} /$ lines per picture width. First-generation intensifiers are devices in which the primary photoelectrons are accelerated by a high electric field to energy around 15 $\mathrm{keV}$ and electrostatically focused onto a P20 phosphor screen. The resolution of the single stage reaches up to $110 \mathrm{lp} / \mathrm{mm}$ with a gain equal to 270 . The photocathode is an S25 with a peak response at about $540 \mathrm{~nm}$, which is the emission wavelength of a P20 phosphor screen of a third-generation tube. This system allows continuous real-time imaging of an eye fundus examination starting from dye injection until a late phase. All phases are recorded on a tape in order to have complete documentation for each patient. In addition, it is possible to view the images through the monitor, making alignment much easier through use of the control lever and giving the patient comfort and eye safety by minimizing the lighting level.

During choroidal examination, the ICG images considered significant by ophthalmologists are addressed through channel B and the cooled chargecoupled device (4). The switch is easy and is quickly made by depressing the shutter button (SB) over the control lever. The fluorescence wavelength selected by a barrier filter $\left(\mathrm{BF}_{\mathrm{B}}\right)$ is optimal for a cooled CCD response (150 mA/W for $\lambda$ at $830 \mathrm{~nm})$. The CCD is used in an integration mode and the angiogram is read out into a data acquisition module and displayed on the monitor.

For this study an imaging device (EEV CCD 0520) 26 by $17 \mathrm{~mm}$ in size, containing 1152 lines with 770 active pixels per line and $22 \mu \mathrm{m}$ center-to-center spacing was used. The EEV CCD 0520 is designed as a CCD frame transfer but operates in a full-frame slow-scan mode. In this mode both imaging and storage sections are used to form one continuous photosensitive region with the signal read out directly through the read-out section. An interesting step in developing a faster acquisition rate for pictures was the use of a binning operation ${ }^{2}$ with a corresponding reduction of resolution.

Slow scan involves an extended charge collection time. The assembly was enclosed in a light-tight aluminum box for the cooled CCD camera to reduce the amount of thermally generated dark current. Consequently, sensitivity and dynamic range are greatly extended; the dark current is 0.1 electron/pixels/s; thus the signal-to-noise ratio is very high and avoids the drawbacks pointed out in the literature. ${ }^{6}$ On this basis the system was able to detect very low light images acquired over an integration time between 60 and $70 \mathrm{~ms}$. The late-phase angiogram images presented were obtained over a larger integration time (up to $90 \mathrm{~ms}$ ) when the increased light intensity from the source was not sufficient to make up for contrast losses.

\section{IMAGE CHARACTERISTICS}

During the acquisition process, the images are recorded with a dynamic range of 16 bits. Let us explain more accurately the considerations that led to the choice of the camera at the planning stage. It is well known that a large dynamic range allows an image to show the intensity variations in the scene it represents better. In our application, due to the low light level of the source and the consequent low level of the fluorescent image, the information extracted needs to span several orders of magnitude, and a digitizing precision commensurate with this dynamic range is needed. 


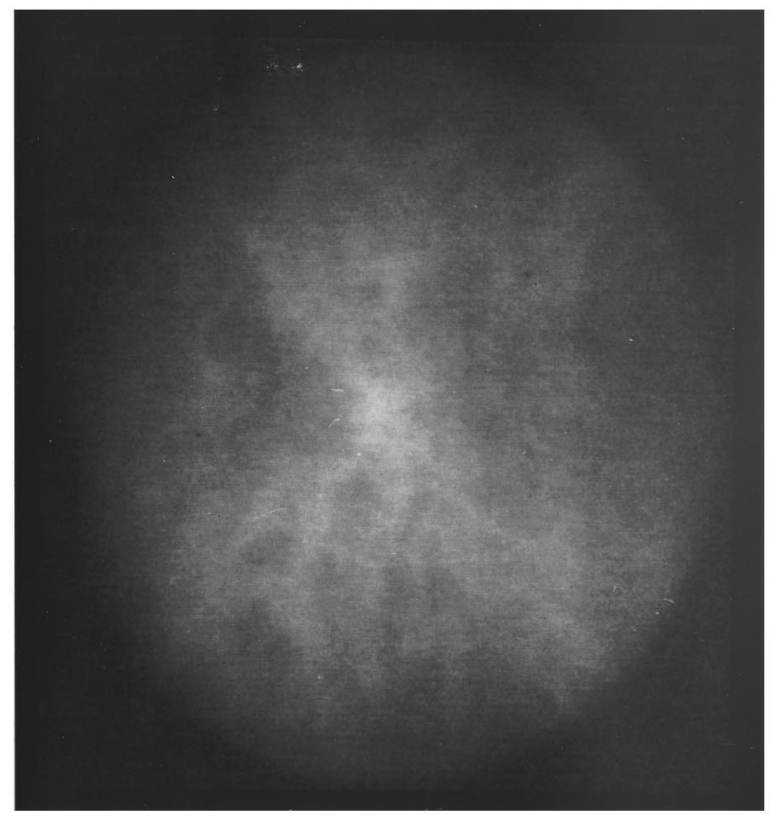

(a)

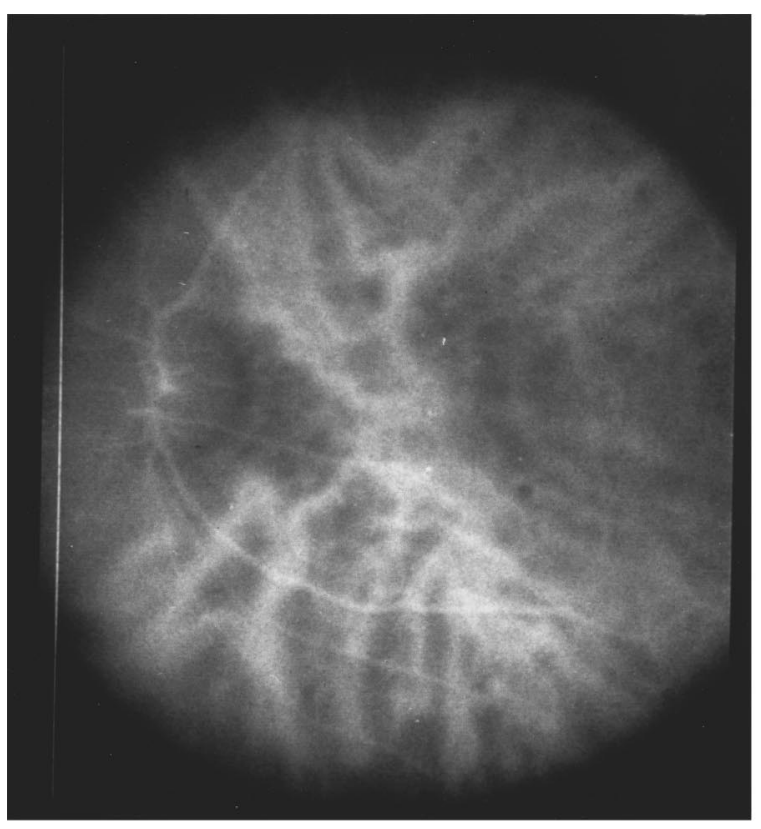

(b)

Fig. 2 Angiogram images recorded 3 min after dye injection. The same angiogram was digitized with 8 bits through channel $A$ (a) and with 16 bits through channel $B(b)$. The image was recorded with an incident irradiance of $300 \mu \mathrm{W} / \mathrm{cm}^{2}$ on the anterior segment.

Figures 2(a) and 2(b) show the same ICG fluorescence image recorded $3 \mathrm{~min}$ after dye injection. The same image was digitized with 8 bits [Figure 2(a)] and 16 bits [Figure 2(b)]. Owing to the very low light level and the consequent poor image dynamics compared with the background noise, only the 16-bit image is able to show the complete network of the choroidal vessels.

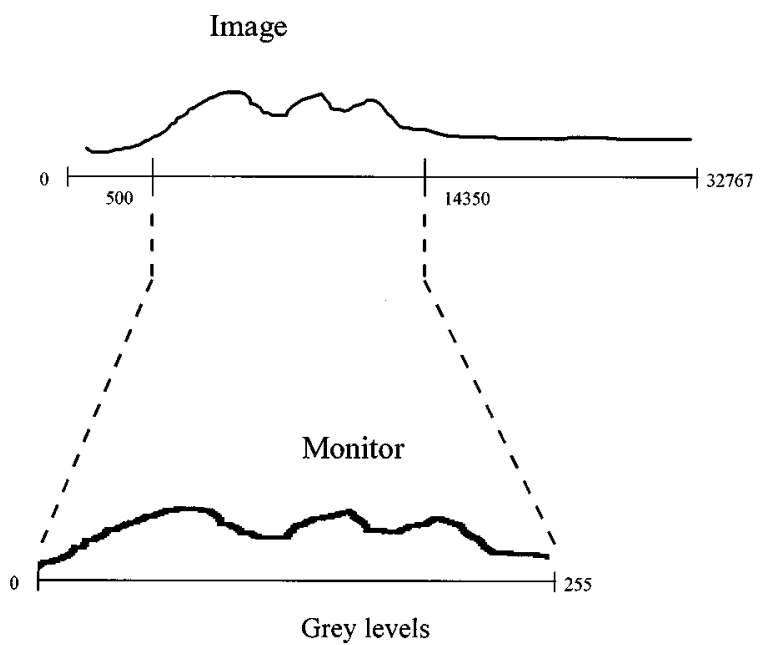

Fig. 3 The gray levels belonging to the range 500 to 14,350 are remapped into the range 0 to 255 .

On the other hand, the usual display adapter has a dynamic range of 8 bits (256 levels) and the monitor has a poorer gray scale. This limits the amount of information that can be displayed at the same time. Moreover, owing to the very low light level dealt with, there is no need to display all the gray levels but only those of interest. Therefore, having such a large dynamic resolution, the end user can exploit all the information contained in the images.

The following example shows the software process applied to the images. For example, in Figure 3 the difference of $14,350-500=13,850$ is the interval of gray with the largest amount of information and is called the window of interest. The end user can easily vary the extremum of this interval and/or enlarge it, in order to show the most interesting details of the image. By means of this procedure, only the significant part of the image is displayed. The observer is then able to get many views of the same image.

It should be noted that in real cases, a monitor shows at the extremum of the luminance some nonlinearities due to the nature of the screen phosphors. This means that an equal difference of gray levels cannot be correctly reproduced. The only way to solve this problem is to modify the gamma curve (parameter describing the nonlinearity of intensity reproduction) of the monitor itself by means of a suitable procedure. A discussion of the meaning and properties of the gamma curve goes beyond the scope of this work, but it should be noted that since the images we are dealing with are characterized by a very low light level, it is important be able to work at all the gray scales.

The visualization process is then composed of two steps: The first one remaps the original graylevel range containing useful information in a narrower (256 levels) range. This is done to avoid waste of memory, disk, and data. The second one, 
with the 8-bit image, has the ability to modify its gamma curve. This is done to improve image contrast. By means of this, the best perceptual aspect can be achieved, increasing, for example, the lower levels of the image and modifying the ratio of light intensity to video signal. Therefore the application of the routines allows one to reach a very high final image quality with a clinician-guided process. In this way, the potential hazard has been reduced without restricting the performance of the instrument.

\section{Methods AND MAterials}

Fundus angiograms were performed in a conventional manner in an outpatient setting with the following modifications:

1. The low-intensity light filtered by the exciting filter was reflected into the patient's eye in order to focus and monitor its fundus by means of the $\mathrm{A}$ channel, which produces a standard video signal for direct viewing on a monitor at $800 \mathrm{~nm}$.

2. After ICG injection, with $\mathrm{BF}_{\mathrm{A}}$ inserted, the whole process was continuously recorded on a tape in real time by the system (3) in the A channel. Angiography was performed using a dye concentration varying from $50 \mathrm{mg} / 2 \mathrm{ml}$ to $50 \mathrm{mg} / 2.5 \mathrm{ml}$. ICG was injected into a peripheral arm vein and was followed immediately by a flush of sterile saline.

3. The same low-intensity lamp was used for both observation and angiography. The ophthalmologist visually evaluated choroidal circulation on the video.

Angiograms that demonstrated atypical features were stored at a resolution of $1152 \times 770$ at 16 bits with a maximum gamma window. Images were stored in real time by depressing the button over the control lever (SB), which deflects the optical path of the fundus image to the cooled camera (4) in the B channel. The integration time varied from $60 \mathrm{~ms}$ for a single angiographic image to $90 \mathrm{~ms}$ for the late-phase images. The images demonstrated no degradation of quality compared with images recorded during previous phases. The minimal acquisition rate of the images with high definition was $1 / 3$ s. By setting a binning factor ${ }^{2}$ of the image equal to 2, early-phase angiograms were performed, reducing the acquisition rate to 1 /s (Figure 4).

Forty-eight patients diagnosed with CSC were evaluated. The age range was 24 to 50 years, with a mean of 34 years; 39 were male and 9 female. The diagnosis of CSC was made by ophthalmoscopy and FA; acute CSC was defined by a serous retinal detachment and a focal fluorescein leakage point, with or without pigment epithelium detachment (PED).

The diagnosis of chronic CSC was made for patients with a previous history of CSC, and retinal pigment epithelium (RPE) alterations were docu-

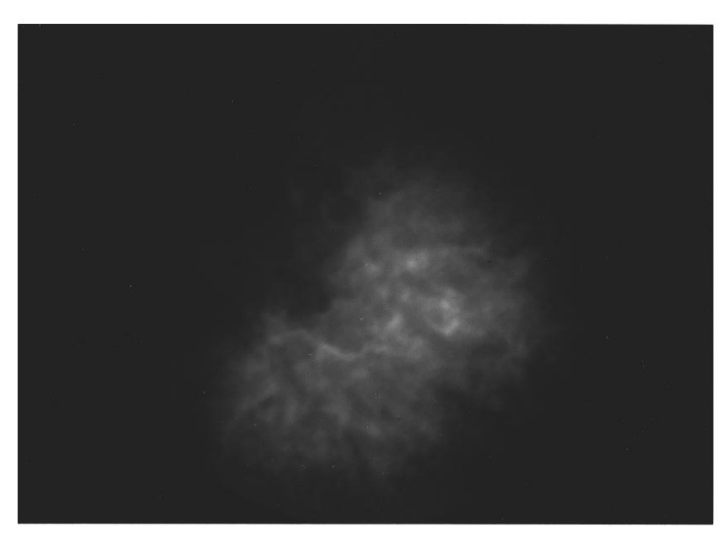

Fig. 4 A very early-phase angiogram (12 s after dye injection).

mented by FA. In this group the active forms were distinguished from the inactive ones because of the presence in the former of acute signs (i.e., serous retinal detachment and fluorescein leakage point) and symptoms of CSC.

Patients were excluded from the study if they also had another macular disorder which would confound interpretation. Patients over 50 were excluded to exclude possible cases of age-related macular degeneration (AMD). Of the patients meeting our criteria, 28 were classified with acute CSC and 20 with chronic CSC. Within the chronic CSC population, 9 cases had active disease and 11 were inactive. Patient examinations included visual acuity, metamorphopsia evaluation by grid testing, ophthalmoscopic examination, FA, and ICGV.

\section{RESUlTS}

A focal subretinal hyperfluorescent leak was shown by FA in all cases (37) of acute and chronic active CSC. ICGV showed a colorizing hyperfluorescent site in 32 cases. The hyperfluorescent foci appeared in early phases (after 2 to $3 \mathrm{~min}$ ) followed by increasing intensity and diffusion in late phases (Figures 5 and 6) similar to that seen with FA. No focal

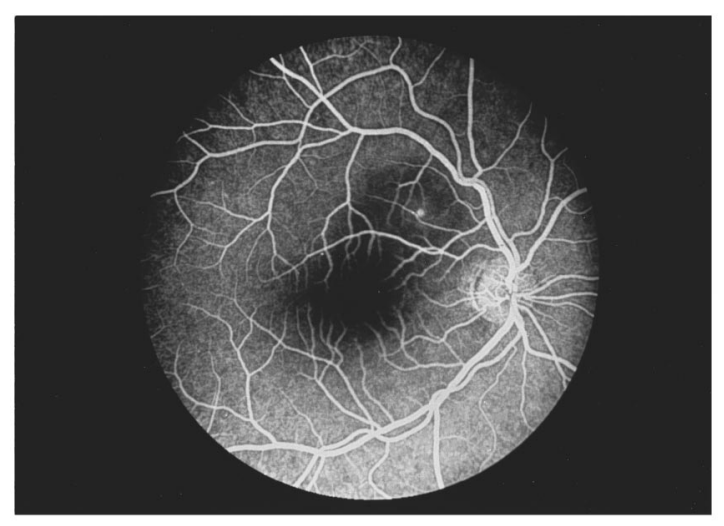

Fig. 5 Fluorescein angiography of the right eye of a 34-year-old patient affected by acute CSC reveals the focal leakage point. 


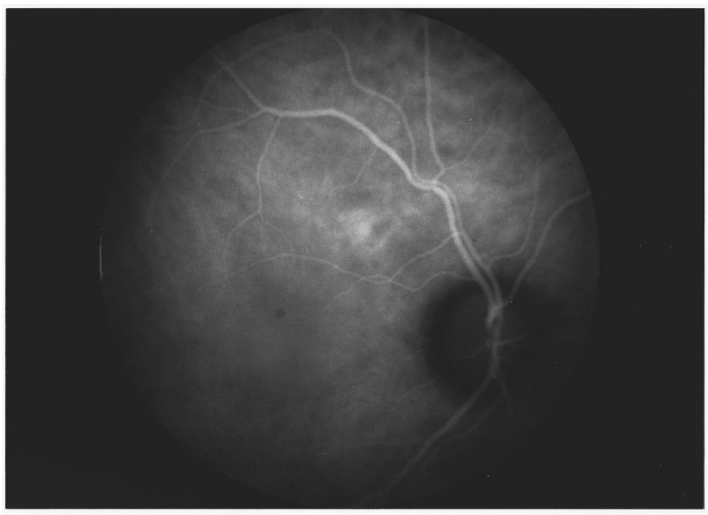

Fig. 6 ICG shows the corresponding leakage point visible in the early phases.

ICG leakage was found in those patients clinically judged to have inactive CSR (chronic).

ICGV also revealed the presence of hyperfluorescent areas that were not evident with FA, appearing 5 to $8 \mathrm{~min}$ after the injection, with a maximum intensity at the 15th minute. We found hyperfluorescent areas in 17 acute CSCs, in 6 active chronic cases, and in 8 inactive cases. More often these hyperfluorescent areas were single and under the focal leak, or under the PED (Figures 7-10). The margins of the hyperfluorescent areas did not correspond to the retinal detachment surrounding the focal leak. In some cases hyperfluorescent areas were found independently from leakage sites or PED and were multiple and diffused in the posterior fundus. PED appeared with ICG as early hyperfluorescent areas showing, in very late phases, a reduction of fluorescence, which usually remained at the PEDS's margins (Figure 11). We found the same number of PEDs with ICG as with FA.

In 15 cases we found a delay in choroidal filling involving the area of focal leakage in active cases and of RPE disorder in inactive cases. We defined choroidal filling delay as a delayed choroidal perfusion evidenced by a hypofluorescent area that was not due to any block effect and that persisted

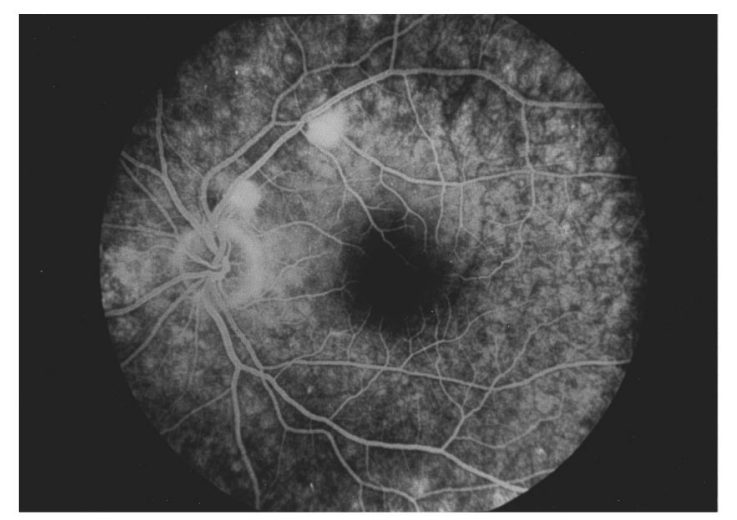

Fig. 7 FA shows a case of acute CSC with an actively leaking point and, near the disk, a PED.

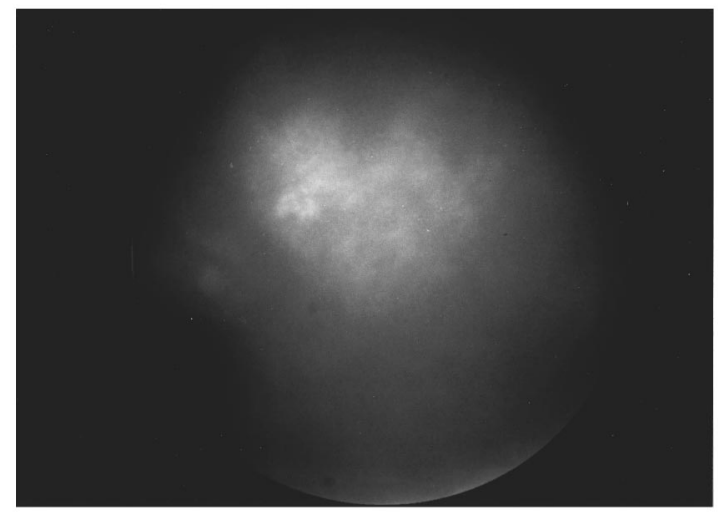

Fig. 8 In late phases ICG shows the PED, still faintly hyperfluorescent. The active leakage point is still visible and is surrounded by a large area of heart-shaped hyperfluorescence.

in the arterial filling phase (Figure 12). FA showed a delay in choroidal filling in 14 cases. In chronic (active and inactive) cases, ICGV revealed little areas of faint hypofluorescence during a full examination that was more visible in the late phase. These areas corresponded to those found by FA and to RPE alterations visible in ophthalmoscopic examination (Figure 13).

\section{DISCUSSION}

The results obtained showed that effort was successfully directed toward minimizing eye exposure and optimizing fundus image quality, allowing the ophthalmologist to make a complete diagnosis. The fundus camera described was developed for use with low lighting for patient comfort. The sensitivity of a cooled CCD camera seems to be sufficient, together with the increased camera integration time, to record both low-intensity sequential angiograms and late-phase ones. Very early phases were recorded with reduced resolution. A sufficiently high signal-to-noise ratio was a necessary condition for accurate angiograms.

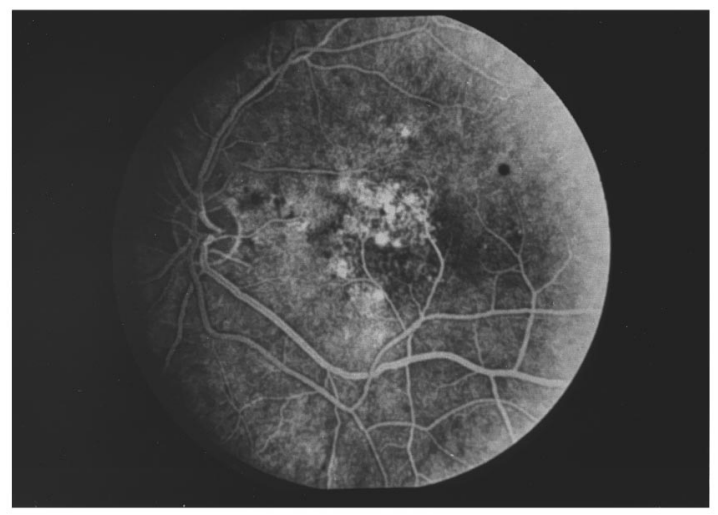

Fig. 9 FA reveals RPE changes and some leakage points in the macular area in this patient with a chronic active CSC. 


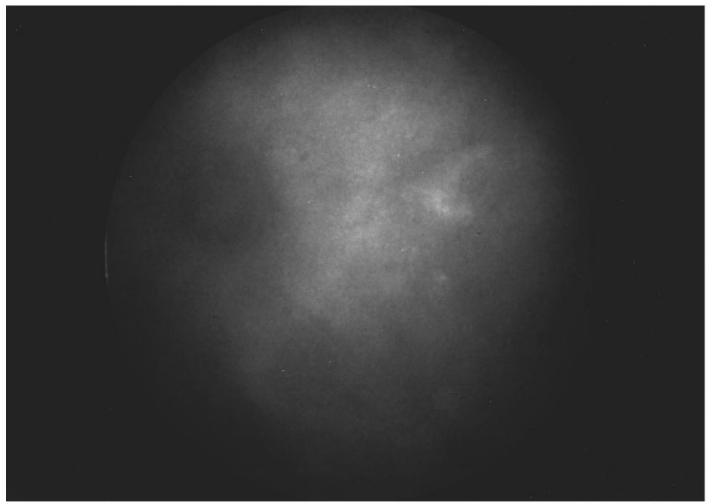

Fig. 10 In the same patient, ICG angiography shows a large area of hyperfluorescence and some areas of hypofluorescence, probably due to the disorder of the RPE.

CSC is a disease typically affecting males between 20 and 50 years of age and is characterized by a decrease in visual acuity, positive central scotoma, and metamorphopsia. The fundus changes are represented by a focal serous detachment of the neuroretinal layer and/or of retinal pigment. The main significant diagnostic tool in the study of CSC is at present represented by FA, showing a typical fluorescein leakage at the level of the RPE with secondary diffusion under the detached retina. Until the advent of digital ICG videoangiography (IGCV), the pathogenesis of CSC was not clear. ${ }^{7-9}$ FA cannot demonstrate whether the permeability alteration primarily involves the choriocapillaris or the RPE, because of the imaging limitations of this technique. These are caused by the blocking effect of the RPE and the physiological leakage of fluorescein from the choriocapillaris, which hides the vascular details in early phases.

The characteristics of ICGV allow us to investigate the choroidal vasculature better, by virtue of the infrared wavelength used, which penetrates and passes through the retinal layers and the RPE (and xantofill). Furthermore, the ICG dye binds al-

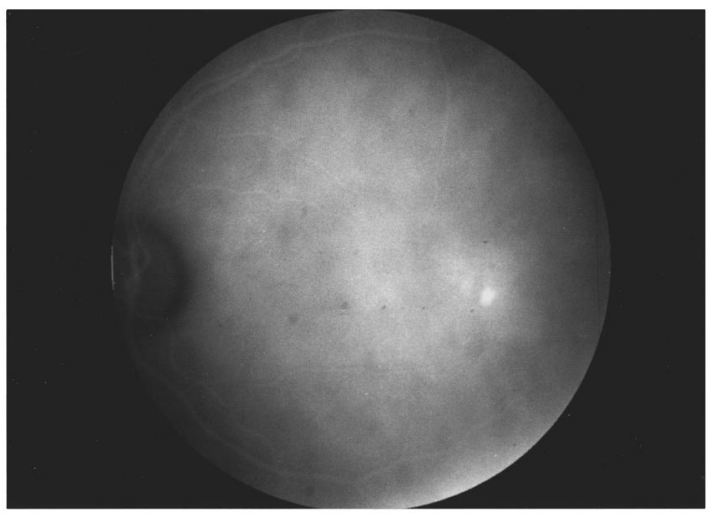

Fig. 11 ICG angiography in early phases $(6 \mathrm{~min})$ shows the serous detachment of the RPE as a hyperfluorescent area temporal to the fovea.

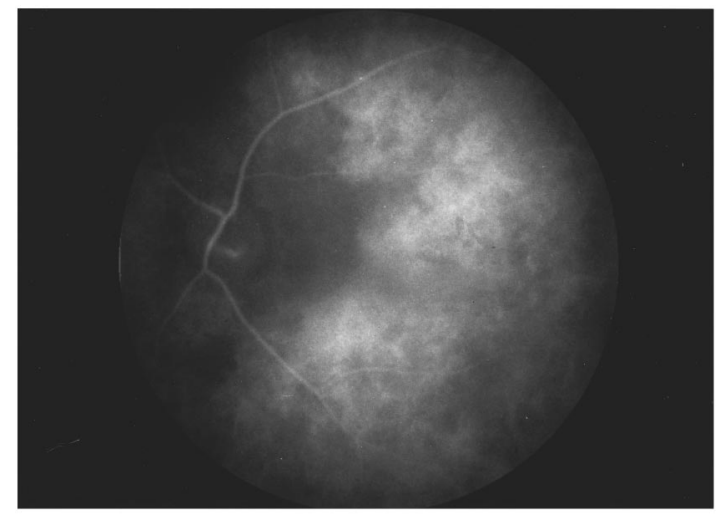

Fig. 12 In this case of CSC with a leakage point near the disk, ICG revealed a delayed choroidal filling pattern involving the interpapillomacular area and the area over the optic disk.

most completely to blood protein, thus preventing a marked leakage from the choriocapillaris. ${ }^{10,11}$ In healthy people ICGV does not reveal hyperfluorescent areas. These areas are present in CSC and are consistent with an alteration of choroidal permeability. We exclude the possibility of their being due to subretinal pooling, because they do not correspond to the margins of the serous retinal detachment.

With FA we cannot see any hyperfluorescent area because the RPE blocks the wavelengths in the visible spectrum, and because fluorescein leaks physiologically from the choriocapillaris. The presence in acute and chronic CSC of hyperpermeable choroidal areas, visible both at the level of exudative points and in the absence of any FA alteration, demonstrates the primary involvement of the choroid in CSC pathogenesis. When accumulation of fluid and proteins in the choroidal district saturates the pump function of the RPE, then fluid passes through the RPE and leaks into the subretinal space. According to this hypothesis, a long-term follow-up would be of great importance in evaluat-

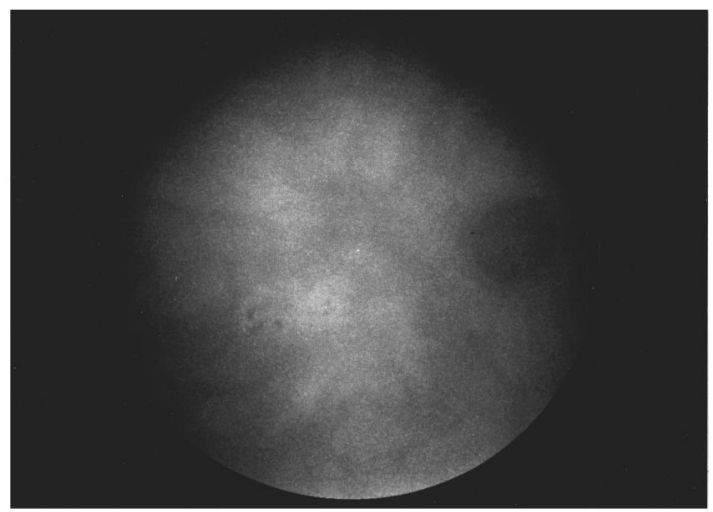

Fig. 13 In a chronic inactive CSC, ICGV shows an area of late choroidal staining and some little areas of hypofluorescence, corresponding to ophthalmoscopically visible RPE alterations. 
ing whether new leakage points form at the level of hyperpermeable areas.

Our results are similar and comparable with findings of other authors who performed ICG angiography, ${ }^{12-17}$ but nevertheless are different. Guyer and co-workers ${ }^{15}$ reported multiple occult serous PEDs found by ICGV, which had not been detected by FA, and hypothesized that a serous retinal pigment epithelium detachment was the first consequence of an abnormal presence of exudate in choroidal space and the first step in CSC. In the present study we did not find occult serous PEDs.

The finding of a delayed choroidal filling is a controversial matter. With LLL videoangiography perfusion, a choroidal deficit was observed in $32 \%$ of cases. These results are similar to our FA findings and agree with other reports. ${ }^{12,13}$ In this study, areas of delayed choroidal filling were indicated by typical signs of CSC, such as focal leakage or RPE alterations, and in most cases presented, with ICGV, a late hyperfluorescence. Such results seem to confirm an ischemic origin of CSC, as proposed by other authors. ${ }^{17}$ However, further study is necessary to clarify this aspect. The aim of the present study was to obtain a system with performance useful for an effective clinical diagnosis using an LLL digital ICGV system.

\section{CONCLUSIONS}

The performance of the current fundus camera can be summarized as follows:

\section{Advantages:}

- use of the same very low-level continuous light source during both observation and imaging;

- ability to continuously record all angiographic phases;

- high dynamic range;

- good sensitivity; the ability to change integration time during different phases allows optimization of image quality; and

- value of signal-to-noise ratio allows the use of all dynamic ranges available.

Disadvantages:

- The acquisition rate of high-definition angiograms is $3 \mathrm{~s}$. This time could be critical for the early phase, compared with the acquisition rate of current systems.

Compared with the scanning laser ophthalmoscope (SLO), the main advantage is high image quality, even with angles of coverage greater than $20 \mathrm{deg}$, and the ability to perform late-phase imaging better. The disadvantage is the lower time resolution; the SLO analyzes the early phases better. Attempts are under way to modify the lighting system in order to further improve the image features.
A good alternative for increasing fundus lighting is the use of an integrating sphere with a near infrared semiconductor laser beam that enters the aperture plane normally. The use of an integrating sphere as a light source is advantageous both for its uniform lighting of the eye fundus and the elimination of the superposition interference effects in glass components in the pathway that are caused by the coherence of a laser beam. The former allows an appropriate use of a high number of gray levels; the latter produces a fine network of laser speckles. This system was tested in the laboratory with very encouraging results. Work is under way to increase the acquisition rate of images mainly for the early phases and experiment with lower tracer concentrations.

\section{REFERENCES}

1. R. W. Flower, "Evolution of indocyanine green dye choroidal angiography," Opt. Eng. 34(3), 727-736 (1995).

2. G. Longobardi, C. Ciamberlini, V. Guarnieri, P. Poggi, G. P. Puccioni, G. Panzardi, and F. Faraldi, "A high sensitivity optoelectronic system for videoangiography," in Proc. SPIE 2084, 157-162 (1994).

3. D. H. Sliney and B. E. Stuck, "Microwave exposure limits for the eye: applying infrared laser threshold data," in $R a$ diofrequency Standard, B. J. Klauenberg, M. Grandolfo, and D. N. Erwin, Eds., pp. 79-87, Plenum Press, New York (1994).

4. D. H. Sliney, "Laser safety," Lasers Surg. Med. 16, 215-225 (1995).

5. R. Pratesi, "Effects of laser radiation on the human tissues," in Proc. Safety in Laser Use, CISE, 5-40 (1994).

6. U. Klingbeil, J. Canter, and M. Lesiecki, "Laser-based fundus camera for infrared angiography," Opt. Eng. 34(3), 737745 (1995)

7. J. D. M. Gass, "Pathogenesis of disciform detachment of the neuroepithelium," Am. J. Ophthalmol. 69, 605-613 (1965).

8. M. Spitznas, "Pathogenesis of central serous retinopathy: a new working hypothesis," Graefes Arch. Clin. Exp. Ophthalmol. 224, 321-324 (1986).

9. M. F. Marmor, "New hypothesis on the pathogenesis and treatment of serous retinal detachment," Graefes Arch. Clin. Exp. Ophthalmol. 226, 548-552 (1988).

10. I. S. Fox and E. H. Wood, "Indocyanine green: physical and pathological properties," Proc. Mayo Clinic. 35, 732-744 (1960).

11. P. M. Bischoff and R. W. Flower, "Ten years experience with choroidal angiography using indocyanine green dye. A new routine examination or an epilogue?," Doc. Ophthalmol. 60, 235-291 (1985).

12. K. Hayashi, Y. Hasegawa, and T. Tokoro, "Indocyanine green angiography of central serous chorioretinopathy," Int. Ophthalmol. 9, 37-41 (1986).

13. A. Scheider, J. E. Nasemann, and O. E. Lund, "Fluorescein and indocyanine green angiographies of central serous choroidopathy by scanning laser ophthalmoscopy," Am. J. Ophthalmol. 115, 50-56 (1993).

14. F. Cardillo Piccolino and L. Borgia, "Central serous chorioretinopathy and indocyanine green angiography," Retina 14(3), 231-242 (1994).

15. D. R. Guyer, L. A. Yannuzzi, and D. Orlock, "Digital indocyanine green videoangiography of central serous chorioretinopathy," Arch. Ophthalmol. 112, 1057-1062 (1994).

16. G. Panzardi, M. C. Donati, F. Faraldi, G. Longobardi, and C. Ciamberlini, "Fluorescein angiography, indocyanine green videoangiography of central serous choroidopathy," in ISFA '94-International Symposium on Fluorescence Angiography, p. 98 (1994).

17. C. Prunte and J. Flammer, "Choroidal capillary and venous congestion in central serous chorioretinopathy," Am. J. Ophthalmol. 121, 26-34 (1996). 